ISSN 1028-821X. Radiofiz. Electron. 2020. Vol. 25, No. 2: 22-28

DOI: https://doi.org/10.15407/rej2020.02.022

УДК 621.396.967;551.510.52

PACS 92.60.H-, 92.60.if, 92.60.Ta, 93.85.-q

\title{
В.А. Кабанов
}

Институт радиофизики и электроники им. А.Я. Усикова НАН Украины

12, ул. Акад. Проскуры, Харьков, 61085, Украина

E-mail:kabanov@ire.kharkov.ua

\section{Капибровка радиометра по относитепьным измерениям яркостной температуры неба под разными угаами места}

Предмет и цель работы. Одним из актуальных направлений дистанционного зондирования окружающей среды является пассивное зондирование атмосферы в СВЧ-диапазоне. Наиболее сложная задача радиометрических измерений - калибровка измерительной системы. Это связано с отсутствием внешних калибровочных элементов, яркостная температура которых известна с высокой точностью. В статье рассматривается способ определения яркостной температуры небосвода, основанный на относительных измерениях под разными углами места. Определенные таким способом уточненные значения яркостной температуры могут быть использованы для калибровки радиометрической системы.

Методы и методология. За основу была взята зависимость яркостной температуры атмосферы от угла места в рамках плоскослоистой изотермической модели атмосферы. Измерения проводились с помощью радиометрической системы трехсантиметрового диапазона с рупорно-параболической антенной, совмещенной с метеолокатором МРЛ-1 и имеющей доступ ко всему диапазону углов места. Кроме того, для измерений из окна здания использовалась совмещенная установка радиометров двух диапазонов (10 и 36 ГГи).

Результаты работы. Проанализирована и опробована предложенная ранее методика калибровки радиометрической системы по температуре небосвода, определенной с помощью относительных измерений под разными углами места. Исследована возможность калибровки при недоступности измерений в зените.

Заключение. Предложенный метод определения температуры небосвода с помощью относительных измерений под разными углами места значительно повымает точность калибровки радиометрической системы и расширяет диапазон погодных условий, при которых такая калибровка допустима. Показана возможность калибровки радиометрической системы по температуре небосвода при недоступности измерений в зените. Ил. 4. Табл. 2. Библиогр.: 5 назв.

Ключевые слова: яркостная температура, радиометр, калибровка, относительные измерения.

Методы радиотеплолокации нашли широкое применение в метеорологии для дистанционного определения различных параметров атмосферы и подстилающей поверхности.

Наиболее сложным моментом радиометрических измерений является калибровка измерительной системы, которая необходима для установления однозначного соответствия между измеряемой яркостной температурой $T_{\text {я иссле- }}$ дуемого объекта и выходным сигналом радиометрического приемника. Калибровка служит основой последующих измерений, от ее качества зависит достоверность получаемых данных.
Калибровка радиометрической системы может выполняться поэлементно: отдельно для антенны и для радиометрического приемника. Но наиболее эффективным методом является внешняя калибровка, так как она учитывает влияние всех возможных факторов на результаты измерений и исключает ошибки, связанные с переключениями на входе радиометра.

В общем случае для реализации качественной калибровки радиометрической системы необходимо иметь набор эталонных излучающих элементов с известной яркостной температурой, перекрывающих весь диапазон ее воз- 
можного изменения для исследуемых объектов. Эти элементы должны быть расположены в дальней зоне антенны и иметь размеры, превышающие размеры пятна главного лепестка диаграммы направленности антенны. Примером такого элемента с известным значением $T_{\text {я }}$ может служить яркостная температура ясного неба в зените $T_{\text {я н }}\left(90^{\circ}\right)[1,2]$.

Такую калибровку практически очень сложно реализовать в связи с тем, что кроме температуры ясного неба трудно найти или организовать другие эталонные элементы с различными яркостными температурами.

В радиометеорологии яркостная температура объектов изменяется в сравнительно небольших пределах $(0 \ldots 350$ К). Если зависимость выходного сигнала радиометра от яркостной температуры близка к линейной, то для калибровки радиометрической системы достаточно

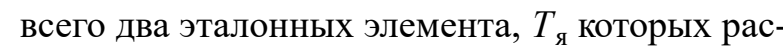
положены вблизи границ указанного диапазона. При этом в роли «холодного» элемента наиболее удобным и доступным является температура ясного неба в зените. В роли «горячего» элемента может использоваться согласованная нагрузка, подключаемая к входу приемника, как это описано в работе [3]. Температуру такой нагрузки легко измерить. В тоже время достаточно просто организовать калибровочный элемент с температурой окружающей среды, которую также легко измерить. Это может быть поверхность, покрытая поглотителем. Можно, наконец, провести измерения внутри помещения с контролируемой температурой. Замкнутое пространство за счет многократного отражения эквивалентно абсолютно черному телу с яркостной температурой, равной термодинамической. В двух последних случаях исключаются ошибки, связанные с переключением входа радиометра между антенной и нагрузкой.

Очевидно, что точность калибровки особенно важна в области малых значений яркостных температур. Следовательно, качество калибровки главным образом определяется точностью оценки температуры ясного неба, которая зависит от поглощения в атмосфере на данный момент времени и может быть определена лишь ориентировочно по статистическим данным, известным из литературных источников, или по результатам собственных измерений.

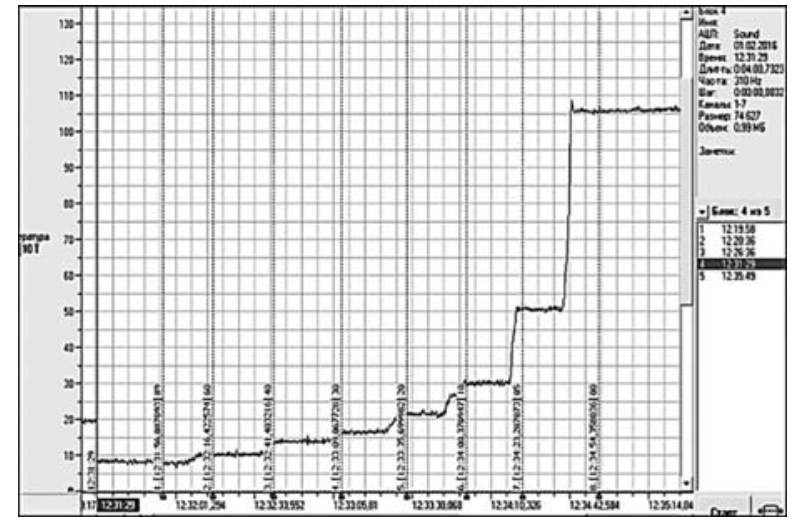

Puc. 1. Пример записи $T_{\text {я н }}$ при разных углах места от зенита до горизонта (слабая облачность)

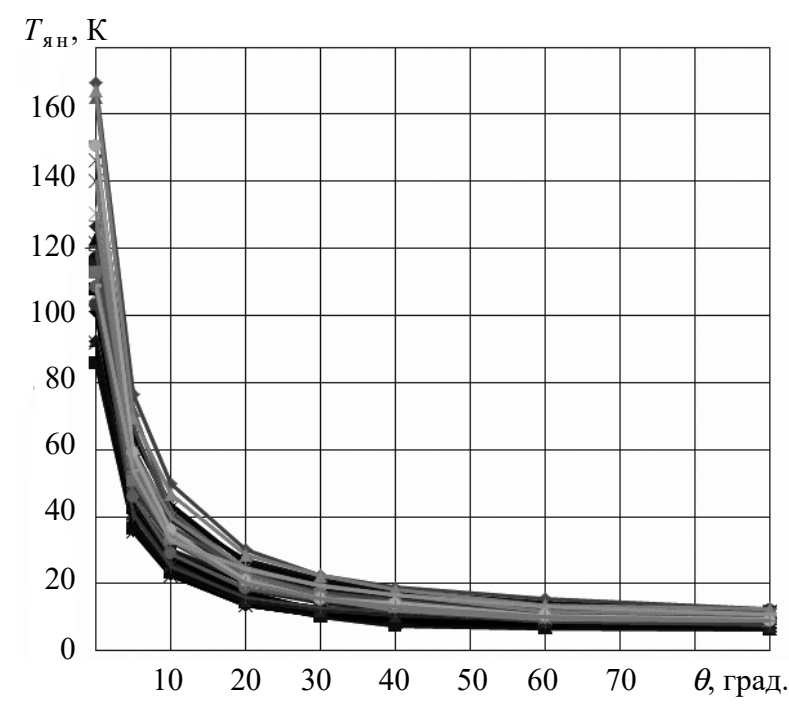

Puc. 2. Набор зависимостей $T_{\text {я н }}(\theta)$ для случаев ясного неба, умеренной облачности и слабого дождя

Согласно [4], яркостная температура неба в зените для трехсантиметрового диапазона даже при дожде интенсивностью 4 мм/час мало отличается от температуры ясного неба. Похожие результаты получены автором в результате измерений в разные сезоны и при различных метеоусловиях.

На рис. 1 приведен пример записи сигнала трехсантиметрового радиометра при углах места $\theta$ от зенита до горизонта. На рис. 2 представлен набор таких зависимостей $T_{\text {я н }}(\theta)$ для случаев ясного неба, умеренной облачности и слабого дождя. Измерения проводились с помощью разработанной автором [3] радиометрической системы с рупорно-параболической антенной, совмещенной с метеолокатором МРЛ-1 (рис. 3). 


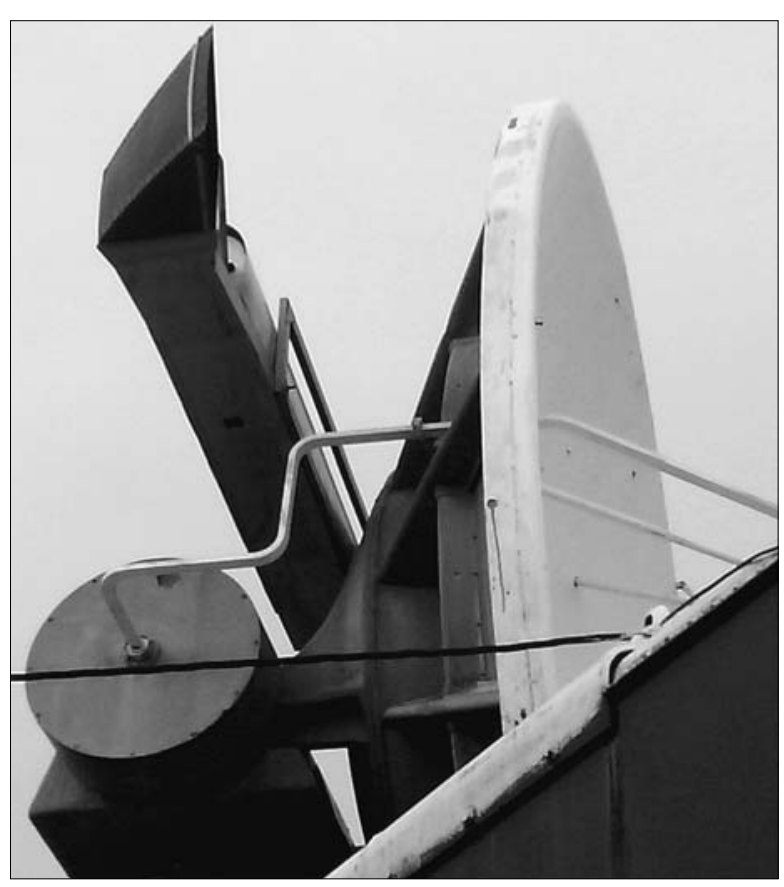

Puc. 3. Расположение радиометра с рупорно-параболической антенной на МРЛ-1

Эти опыты показали относительную стабильность $T_{\text {я н }}\left(90^{\circ}\right)$. Так, при минимальном значении, равном $6,5 \mathrm{~K}$, среднее значение (если исключить случаи сильных аномалий, например таких, как грозовые облака или интенсивные дожди) оказалось равным 9,3 К.

Следовательно, использование для калибровки трехсантиметрового радиометра значения $T_{\text {я н }}\left(90^{\circ}\right)$ примерно $9 \mathrm{~K}$ в большинстве случаев (кроме резко аномальных) дает погрешность калибровки $2 \ldots 3 \mathrm{~K}$.

Точность такой калибровки вполне приемлема при исследовании объектов с большими яркостными температурами, однако она недостаточна для анализа безоблачной и малооблачной атмосферы. Кроме того, при сплошной облачности трудно оценить достоверность калибровки.

Существенно повысить точность калибровки и расширить диапазон погодных условий, при которых такая калибровка допустима, можно с помощью относительных измерений яркостной температуры небосвода $T_{\text {я н }}$ под разными углами места. Сделать это можно следующим образом. Яркостная температура небосвода $T_{\text {я н }}$ складывается из температуры космического излучения $T_{\text {я к }}$ (реликтовое излучение) и излучения, связанного с поглощением в aтмосфере $T_{\text {я ат }}$ :

$T_{\text {я н }}=T_{\text {я к }}+T_{\text {я ат }}$.

Температура космического излучения равна 2,72 К в широком диапазоне частот [5]. При значительном увеличении поглощения в атмосфере и росте яркостной температуры вклад космического излучения падает и его можно не учитывать. Как правило, в этом случае снижаются и требования к абсолютной точности калибровки.

Значение $T_{\text {я ат зависит от поглощения атмо- }}$ сферой в направлении визирования антенны и средней термодинамической температуры атмосферы $T_{\text {ср ат }}$ В свою очередь, поглощение зависит от основных метеопараметров атмосферы и толщины поглощающего слоя.

Если воспользоваться приближением плоскослоистой изотермической модели атмосферы, то для наземного радиометра толщина поглощающего слоя зависит от угла места и равна толщине в зените, деленной на $\sin \theta$. Плоскослоистая модель справедлива для углов $\theta$ от 5 до $90^{\circ}$.

Зависимость яркостной температуры атмосферы от угла места $\theta$ и полного вертикального поглощения $\tau$ имеет вид:

$T_{\text {я ат }}(\theta)=T_{\text {ср ат }}\left(1-e^{\tau / \sin \theta}\right)$,

где $\tau=\ln \left(1-T_{\text {я ат }}\left(90^{\circ}\right) / T_{\text {ср ат }}\right) ; T_{\text {ср ат }}-$ средняя температура атмосферы. Принято считать, что $T_{\text {ср ат }}=\left(T_{0 \text { ат }}-32\right) \mathrm{K}$, где $T_{0 \text { ат }}-$ температура у поверхности Земли.

В общем случае зависимость $T_{\text {я ат }}$ от толщины поглощающего слоя носит нелинейный характер в связи с уменьшением вклада в яркостную температуру более удаленных участков атмосферы из-за поглощения излучения на пути к антенне радиометра. Однако при малых значениях поглощения в атмосфере этой нелинейностью можно пренебречь и записать простое выражение для яркостной температуры

$T_{\text {я ат }}(\theta)=T_{\text {я ат }}\left(90^{\circ}\right) / \sin \theta$,

где $T_{\text {я ат }}\left(90^{\circ}\right)-$ соответствует тепловому излучению атмосферы в зените.

Измерив $T_{\text {я н }}$ под двумя разными углами и использовав приведенное выше выражение, можно определить практически точное значе- 
ние $T_{\text {я н }}$ в зените или другом угле $\theta$. Следует отметить, что относительные измерения всегда более точны по сравнению с абсолютными.

Калибровка по уточненной температуре небосвода впервые использовалась для радиометрической системы трехсантиметрового диапазона [3].

Наиболее удобным вариантом являются измерения в зените и под углом места $\theta=30^{\circ}$, когда толщина эффективно поглощающего слоя атмосферы вдвое больше и, следовательно, $T_{\text {я ат }}\left(90^{\circ}\right)$ вдвое выше, чем в зените. Следовательно, разность измеренных под этими углами температур будет равна яркостной температуре атмосферы в зените $T_{\text {я ат }}\left(90^{\circ}\right)$, а двойное значение разности будет соответствовать $T_{\text {я ат }}\left(30^{\circ}\right)$. Добавив к этим величинам температуру космического излучения (с поправкой на поглощение), мы получим точные значения $T_{\text {я н }}\left(90^{\circ}\right)$ и $T_{\text {я н }}\left(30^{\circ}\right)$, которые могут быть использованы для калибровки радиометра.

Уточненные данные можно использовать не только для калибровки радиометра при новых измерениях, но и внести поправки в результаты измерений, выполненных ранее.

При недоступности измерений в зените можно использовать относительные измерения $T_{\text {я н }}$ под другими углами. Такая калибровка применялась при измерениях на разработанной автором совмещенной установке радиометров двух диапазонов (10 и 36 ГГц) из окна здания (рис. 4).

Отметим, что в таких условиях измерений важно иметь антенны с малым уровнем боковых лепестков. Даже рупорные антенны, которые обычно используются как эталонные, имеют ограничения из-за высокого уровня первых боковых лепестков в плоскости $E$. Поэтому для обоих диапазонов были использованы рупорнопараболические антенны.

В качестве примера приведем расчет калибровочных значений $T_{\text {ян }}$ по измерениям под углами 30 и $60^{\circ}$ для радиометра диапазона 36 ГГц. Значения яркостных температур, измеренных радиометром под этими углами, соответственно равны 68,3 К и 46,3 К, а их разность составляет $22 \mathrm{~K}$.

В соответствии с выражением (2), $T_{\text {я ат }}\left(30^{\circ}\right)=$ $=2 T_{\text {я ат }}\left(90^{\circ}\right)$, a $T_{\text {я ат }}\left(60^{\circ}\right)=1,155 T_{\text {я ат }}\left(90^{\circ}\right)$. После несложных преобразований можно записать $T_{\text {я ат }}\left(90^{\circ}\right)=\left[T_{\text {я ат }}\left(30^{\circ}\right)-T_{\text {я ат }}\left(60^{\circ}\right)\right] \cdot 1,183$,

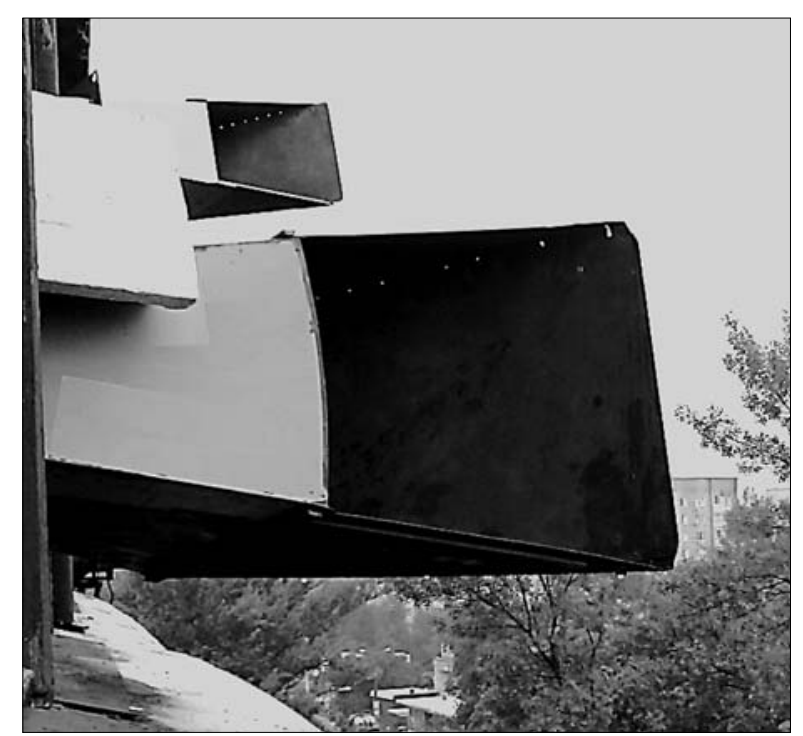

Puc. 4. Расположение системы радиометров с рупорнопараболическими антеннами в окне здания

а в нашем случае $22 \cdot 1,183=26$. При этом $T_{\text {я ат }}\left(30^{\circ}\right)=52 \mathrm{~K}$, а $T_{\text {я ат }}\left(60^{\circ}\right)=30 \mathrm{~K}$. Добавив к этим значениям вклад космического излучения, получим две точки небосвода с известными яркостными температурами, которые соответственно относятся к положению антенны $\theta=30^{\circ}$ и $\theta=60^{\circ}$. Полученные значения $T_{\text {я н могут слу- }}$ жить калибровочными точками для радиометрической системы указанного диапазона.

Результаты измерений и расчетов $T_{\text {ян }}$ для трехсантиметрового радиометра, установленного на МРЛ-1 и имеющего доступ ко всему диапазону углов места, приведены в табл. 1 . В левой части таблицы показаны измеренные значения $T_{\text {я н }}$ под разными углами, а в правой части - рассчитанные $T_{\text {я н }}\left(90^{\circ}\right)$ с использованием углов места 30 и $90^{\circ}$, а также 30 и $60^{\circ}$.

Как видно из таблицы, расчетные уточненные значения $T_{\text {я н }}\left(90^{\circ}\right)$ могут отличаться от измеренных на $5 \ldots 6^{\circ}$. При этом значения $T_{\text {я н }}\left(90^{\circ}\right)$, определенные по разным парам углов, отличаются незначительно. Это говорит о возможности калибровки радиометрической системы при недоступности измерений в зените.

Все сказанное выше справедливо для небольших значений яркостных температур, как это имело место при калибровке радиометра в работе [3].

При увеличении поглощения в атмосфере и повышении яркостной температуры, особенно при использовании более высокочастотных 
Таблиияа 1

\begin{tabular}{|c|c|c|c|c|c|c|}
\hline \multirow{3}{*}{ Дата } & \multirow{3}{*}{ Время } & \multicolumn{5}{|c|}{ Угол места $\theta^{\circ}$} \\
\hline & & $30^{\circ}$ & $60^{\circ}$ & $90^{\circ}$ & $\begin{array}{c}\text { Для углов } \\
(30,90)\end{array}$ & $\begin{array}{c}\text { Для углов } \\
(30,90)\end{array}$ \\
\hline & & \multicolumn{3}{|c|}{$T_{\text {ян }} *$} & \multicolumn{2}{|c|}{$T_{\text {я н }}\left(90^{\circ}\right)^{* *}$} \\
\hline 25.11 .14 & $13: 00$ & 10,77 & 8,54 & 6,77 & 6,70 & 5,34 \\
\hline 27.11 .14 & $11: 30$ & 11,57 & 7,31 & 6,96 & 7,31 & 7,74 \\
\hline 01.12 .14 & $11: 40$ & 10,99 & 7,33 & 6,83 & 6,86 & 7,03 \\
\hline 03.12 .14 & $12: 15$ & 9,83 & 6,16 & 5,84 & 6,69 & 7,04 \\
\hline 08.12 .14 & 13:00 & 15,60 & 8,18 & 6,58 & 11,72 & 11,48 \\
\hline 09.12 .14 & $11: 40$ & 15,63 & 8,76 & 6,40 & 11,93 & 10,83 \\
\hline 10.12 .14 & $12: 00$ & 10,96 & 7,30 & 6,44 & 7,22 & 7,03 \\
\hline 16.12 .14 & $12: 10$ & 11,31 & 8,18 & 7,45 & 6,56 & 6,40 \\
\hline 17.12 .14 & $11: 50$ & 11,77 & 7,19 & 5,86 & 8,61 & 8,12 \\
\hline 02.02 .15 & $12: 00$ & 11,67 & 7,29 & 6,58 & 7,79 & 7,88 \\
\hline 24.03 .15 & $15: 30$ & 12,25 & 8,54 & 8,47 & 6,48 & 7,09 \\
\hline 01.04 .15 & 13:00 & 9,32 & 6,42 & 4,67 & 7,35 & 6,13 \\
\hline 08.04 .15 & $12: 00$ & 13,71 & 5,68 & 5,23 & 11,18 & 12,20 \\
\hline 14.04 .15 & $12: 30$ & 11,70 & 7,26 & 6,57 & 7,83 & 7,95 \\
\hline 09.06 .15 & $11: 00$ & 16,87 & 9,90 & 8,58 & 10,99 & 10,95 \\
\hline 10.06 .15 & $11: 00$ & 15,35 & 10,11 & 8,89 & 9,16 & 8,90 \\
\hline 15.06 .15 & $10: 40$ & 16,14 & 10,45 & 8,03 & 10,81 & 9,43 \\
\hline 17.06 .15 & $11: 30$ & 16,83 & 11,09 & 10,53 & 9,00 & 9,49 \\
\hline 22.06 .15 & $12: 00$ & 22,73 & 17,01 & 15,42 & 10,01 & 9,47 \\
\hline 30.06 .15 & $11: 30$ & 20,11 & 14,36 & 12,97 & & \\
\hline 14.07 .15 & $10: 50$ & 15,97 & 10,32 & 8,34 & 10,33 & 9,38 \\
\hline 14.07 .15 & $12: 00$ & 21,04 & 15,38 & 13,31 & 10,43 & 9,40 \\
\hline 26.10 .15 & $11: 20$ & 10,89 & 6,48 & 5,80 & 7,79 & 7,92 \\
\hline 09.11 .15 & $11: 10$ & 14,73 & 9,52 & 8,52 & 8,91 & 8,86 \\
\hline 10.11 .15 & $11: 00$ & 19,50 & 12,16 & 10,93 & 11,27 & 11,38 \\
\hline 10.11 .15 & $15: 30$ & 16,13 & 9,12 & 8,78 & 10,05 & 10,99 \\
\hline 10.11 .15 & $15: 45$ & 12,87 & 6,76 & 6,54 & 9,03 & 9,93 \\
\hline 10.11 .15 & $15: 52$ & 18,22 & 11,19 & 8,33 & 12,59 & 11,02 \\
\hline 18.11 .15 & $11: 20$ & 16,68 & 10,12 & & 9,71 & 10,46 \\
\hline 19.11 .15 & $11: 15$ & 23,11 & 14,43 & 13,29 & 12,52 & 12,97 \\
\hline 19.11 .15 & $14: 55$ & 16,08 & 10,29 & 9,70 & 9,08 & 9,55 \\
\hline 23.11 .15 & $12: 10$ & 17,37 & 9,94 & 9,09 & 10,98 & 11,49 \\
\hline 30.11 .15 & $11: 20$ & 14,14 & 8,97 & 7,64 & 9,20 & 8,82 \\
\hline 14.12 .15 & $11: 40$ & 14,76 & 9,43 & 8,37 & 9,09 & 9,01 \\
\hline 01.02 .16 & $12: 30$ & 16,49 & 10,31 & 8,14 & 11,05 & 10,01 \\
\hline 05.09 .16 & $11: 45$ & 15,20 & 10,80 & 9,80 & 8,10 & 7,91 \\
\hline 13.09 .16 & $11: 20$ & 11,90 & 6,92 & 6,47 & 8,13 & 8,59 \\
\hline 21.09 .16 & $13: 12$ & 19,58 & 14,65 & 13,48 & 8,80 & 8,53 \\
\hline 26.09 .16 & $15: 40$ & 18,63 & 13,23 & 11,69 & 9,64 & 9,09 \\
\hline \multirow[t]{3}{*}{02.10 .18} & $13: 40$ & 15,99 & 10,06 & 8,96 & 9,73 & 9,72 \\
\hline & & & & & Ср. знач. & Ср. знаг \\
\hline & & & & & 9,26 & 9,13 \\
\hline
\end{tabular}

* - результаты измерений; ** - результаты расчетов.
Таблица 2

\begin{tabular}{|c|c|c|c|c|c|c|}
\hline \multirow{2}{*}{$\theta$} & \multicolumn{5}{|c|}{ Яркостная температура атмосферы $T_{\text {я ат, }}$ К } \\
\cline { 2 - 7 } & $90^{\circ}$ & $60^{\circ}$ & $50^{\circ}$ & $40^{\circ}$ & \multicolumn{1}{c|}{$30^{\circ}$} & \multicolumn{1}{c|}{$20^{\circ}$} \\
\hline Форм. (1) & 10,00 & 11,51 & 12,98 & 15,40 & 19,63 & 28,21 \\
Форм. (2) & 10,00 & 11,55 & 13,05 & 15,56 & 20,00 & 29,24 \\
Форм. (1) & 30,00 & 34,33 & 38,48 & 45,21 & 56,67 & 78,66 \\
Форм. (2) & 30,00 & 34,64 & 39,16 & 46,67 & 60,00 & 87,71 \\
Форм. (1) & 60,00 & 68,01 & 75,52 & 87,37 & 106,67 & 140,51 \\
Форм. (2) & 60,00 & 69,28 & 78,32 & 93,34 & 120,00 & 175,43 \\
\hline
\end{tabular}

диапазонов, погрешность, связанная с использованием упрощенной формулы (2), возрастает. В этих случаях необходимо применять выражение (1). При этом разность значений яркостных температур под разными углами будет выражаться показательными уравнениями, не имеющими общего метода решения. Это усложняет использование более точной формулы.

Можно предложить достаточно простой способ решения этой проблемы. Для этого необходимо составить excel-таблицу, в которой при введении значения $T_{\text {я ат }}\left(90^{\circ}\right)$ автоматически по приведенной формуле рассчитываются $T_{\text {я ат }}$ для необходимых углов места и разности значений для этих углов. Затем методом подбора величины $T_{\text {я ат }}\left(90^{\circ}\right)$ добиваемся совпадения расчетной разности значений для двух углов с измеренной радиометром. Таким образом, находим но-

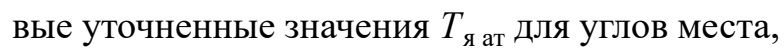
которые могут использоваться для калибровки радиометра. Как показал опыт, этот процесс занимает немного времени и обеспечивает простое применение более точной формулы.

При большом расхождении измеренных и рассчитанных значений $T_{\text {я н }}$ такую калибровку следует повторить с новыми значениями, измеренными после первой калибровки.

Таблица 2 позволяет определить, в каких случаях можно использовать упрощенный расчет по формуле (2), а в каких необходимо использовать более точную формулу (1). В таблице представлен расчет яркостных температур атмосферы по двум формулам для трех значений температур в зените.

При температуре в зените $10 \mathrm{~K}$ различия в расчетах по двум формулам несущественны и можно использовать упрощенную формулу (2). При $T_{\text {я ат }}\left(90^{\circ}\right)$ в пределах $30 \mathrm{~K}$ ошибки находятся в разумных пределах и допустимо использо- 
вание обеих формул, а при более высоких температурах необходимо использовать более точную формулу (1).

Выводы. Предложенный метод калибровки с использованием относительных измерений под разными углами места для определения яркостной температуры небосвода значительно повышает точность калибровки и расширяет диапазон погодных условий, при которых она применима. Благодаря такой калибровке расширяются возможности трехсантиметрового радиометра, позволяя измерять поглощение в атмосфере, составляющее сотые доли децибела, и исследовать параметры даже безоблачной атмосферы.

Появляется возможность калибровки радиометрической системы по температуре небосвода при недоступности измерений в зените.

\section{БИБЛИОГРАФИЧЕСКИЙ СПИСОК}

1. Михайлов В.Ф., Брагин И.В., Брагин С.И. Микроволновая спутниковая аппаратура дистанционного зондирования Земли: учеб. пособие для вузов. Санкт-Петербург: СПбГУАП, 2003. 404 с.

2. Мельник Ю.А., Зубкович С.Г., Степаненко В.Д. Радиолокационные методы исследования Земли. Под ред. Ю.М. Мельника. Москва: Сов. радио, 1980. 264 с.

3. Кабанов В.А. Радиометр для метеорологических измерений с точной калибровкой по яркостной температуре неба. Радиофизика и электроника. 2016. Т. 7(21), № 3. C. 11-17. DOI: https://doi.org/10.15407/rej2016.03.011.

4. Справочник по радиолокаичии: в 4-х т. Т.4. Под ред. М. Сколника. Пер. с англ. Под ред. К.Н. Трофимова. Москва: Сов. радио, 1978. $376 \mathrm{c}$.

5. Зельдович Я.Б., Новиков И.Д. Релятивистская астрофизика. Москва: Наука, 1967. 656 с.

Стаття надійшла 17.09.2019

\section{REFERENCES}

1. Mikhaylov, V.F., Bragin, I.V., Bragin, S.I., 2003. Microwave satellite equipment for remote Earth sensing: manual for graduate students. St. Petersburg: Saint Petersburg State University of Aerospace Instrumentation Publ. (in Russian).

2. Mel'nik, Yu.A., Zubkovich, S.G., Stepanenko, V.D., 1980. Radar methods of Earth exploration. Moscow: Sov. Radio Publ. (in Russian).

3. Kabanov, V.A., 2016. A radiometer for meteorological measurements with accurate calibration against the sky brightness temperature. Radiofiz. Elektron., 7(21)(3), pp. 11-17. DOI: https://doi.org/10.15407/rej2016.03.011.

4. Skolnik, M. ed., 1978. Reference book on radar. Vol. 4. Translated from English and ed. by K.N. Trofimov. Moscow: Sov. Radio Publ. (in Russian).

5. Zel’dovich, Ya.B., Novikov, I.D., 1967. Relativistic astrophysics. Moscow: Nauka Publ. (in Russian).

Received 17.09.2019

V.A. Kabanov

O.Ya. Usikov IRE of NASU

12, Akad. Proskura St., Kharkiv, 61085, Ukraine

RADIOMETER CALIBRATION UPON RELATIVE MEASUREMENTS OF SKY BRIGHTNESS TEMPERATURE AT DIFFERENT ELEVATION ANGLES

Subject and purpose. One of the relevant areas of remote sensing of the environment is the development of microwave methods of passive sensing of the atmosphere. The biggest challenge occurring in radiometric measurements is measuring system calibration. The reason lies in the lack of external calibration elements whose brightness temperatures are known with a high accuracy. The paper deals with the method for determining the sky brightness temperature by relative measurements at different elevation angles. The brightness temperatures refined in the way fit for radiometric system calibration.

Methods and methodology. Basically, the approach is governed by the dependence of the atmospheric brightness temperature on the elevation angle in terms of the plane-layered isothermal atmospheric model. The measurements rely on a three-centimeter radiometric system having a horn-reflector antenna combined with the MPL-1 weather radar, the entire range of elevation angles being accessible. Besides, a combined setup of two, $10 \mathrm{GHz}$ and $36 \mathrm{GHz}$, radiometers is involved for measurements from a building window.

Results. The previously proposed methodology for radiometric system calibration against the sky temperature obtained by relative measurements at different elevation angles has been analyzed and tested. The calibration possibility has been examined in the case that zenith measurements are unavailable.

Conclusion. The proposed method for getting sky temperatures upon relative measurements at different elevation angles significantly increases accuracy of radiometric system calibration and extends a range of weather conditions admitting calibration of the kind. It has been shown that the radiometric system calibration against the sky temperature is possible even with zenith measurements excluded.

Key words: brightness temperature, radiometer, calibration, relative measurements. 
В.О. Кабанов

Інститут радіофізики та електроніки ім. О.Я. Усикова НАН України

12, вул. Акад. Проскури, Харків, 61085, Україна

КАЛІБРУВАННЯ РАДІОМЕТРА ЗА ВІДНОСНИМИ ВИМІРЮВАННЯМИ

ТЕМПЕРАТУРИ ЯСКРАВОСТІ НЕБА ПІД РІЗНИМИ КУТАМИ МІСЦЯ

Предмет і мета роботи. Одним з актуальних напрямків дистанційного зондування навколишнього середовища є пасивне зондування атмосфери у НВЧ-діапазоні. Найбільш складним завданням радіометричних вимірювань $є$ калібрування вимірювальної системи. Це пов'язано з відсутністю зовнішніх калібрувальних елементів, яскравісна температура яких відома з високою точністю. У статті розглядається спосіб визначення яскравісної температури небосхилу, заснований на відносних вимірюваннях під різними кутами місця. Визначені у такий спосіб уточнені значення температури яскравості можуть бути використані для калібрування радіометричної системи.

Методи і методологія. За основу було покладено залежність яскравісної температури атмосфери від кута місця в рамках плоскошаруватої ізотермічної моделі атмосфери. Вимірювання проводилися за допомогою радіометричної системи трисантиметрового діапазону з рупорно-параболічною антеною, яка суміщена з метеолокатором МРЛ-1 і має доступ до всього діапазону кутів місця. Крім того, для вимірювань з вікна будівлі було застосовано поєднану установку радіометрів двох діапазонів (10 і 36 ГГц).

Результати роботи. Проаналізовано і випробувано запропоновану раніше методику калібрування радіометричної системи за температурою небосхилу, яку визначено за допомогою відносних вимірювань під різними кутами місця. Досліджено можливість калібрування у випадку недоступності вимірювань у зеніті.

Висновок. Запропонований метод визначення температури небосхилу за допомогою відносних вимірювань під різними кутами місця значно підвищує точність калібрування радіометричної системи і розширює діапазон погодних умов, при яких таке калібрування допустиме. Показано можливість калібрування радіометричної системи за температурою небосхилу у випадку недоступності вимірювань у зеніті.

Ключові слова: яскравісна температура, радіометр, калібрування, відносні вимірювання. 